\title{
EFECTO DE LA GnRH EN EL PROCESO DEL RECONOCIMIENTO MATERNAL DE LA PREÑEZ SOBRE LA SUPERVIVENCIA EMBRIONARIA EN ALPACAS
}

\author{
Mariluz Araínga R. ${ }^{1}$, Víctor Leyva V. ${ }^{2}$, Wilber García V. ${ }^{3}$ y Enrique Franco Ll. ${ }^{3}$
}

\section{ABSTRACT}

\begin{abstract}
The effect of GnRH administered around the time of maternal recognition of prenancy on the embryonic survival rate was studied. It was used 67 adult female alpacas sexually receptive to the male, and bearing a preovulatory follicle $\geq 7 \mathrm{~mm}$, detected by rectal ultrasonography. Animals were distributed in three groups: $\mathrm{G} 0(\mathrm{n}=23)$ as control; $\mathrm{G} 1$ $(\mathrm{n}=22)$ received $6 \mu \mathrm{g}$ GnRH on day 4 after ovulation; and $\mathrm{G} 2(\mathrm{n}=22)$ received $4 \mu \mathrm{g} \mathrm{GnRH}$ on days 8 and 9 after ovulation. The ocurrence of ovulation was confirmed through the detection of a corpus luteum (CL) on day 5 after ovulation. Fertilization and gestation viab-ility were monitored by CL development, structural changes of the uterine horns and presence of the embryonic vesicle on days 12, 18, 25 and 30 after ovulation, using rectal ultrasonography. The female sexual receptivity to the male was simultaneously evaluated. The number of females that ovulate was 20, 22 and 21 in G0, G1, and G2 respectively, and was presumed that conception has occurred. However, on day 12, the embryo survival rate was $90.5 \%$ in $\mathrm{G} 1,75.0 \%$ in $\mathrm{G} 0$, and $76.2 \%$ in $\mathrm{G} 2$. These values were statistically different $(\mathrm{p}<0.05)$ on day 25 and 30 between $\mathrm{G} 1(76.2 \%)$ and $\mathrm{G} 2(42.9 \%)$. The follicle diameter in $\mathrm{G} 2$ showed a continuos decrease from day 0 to day 5 and 12 after ovulation (7.4, 7.3 and $6.3 \mathrm{~mm}$, respectively). The CL size of females that maintained pregnancy showed an increase from day 5 to day 12 and 18 after ovulation, while those of females that had embryonic mortality showed a significant $(\mathrm{p}<0.01)$ decrease since day 12 onwards. There was an inverse relationship between the diameter of CL and follicle size with embryonic mortality. It is concluded that $\mathrm{GnRH}$ decreased the size of the dominant follicle and embryo survival rate when applied around the time of maternal recognition of pregnancy.
\end{abstract}

Key words: GnRH, alpaca, embryonic mortality

\section{RESUMIEN}

El efecto de la GnRH sobre el porcentaje de supervivencia embrionaria durante el periodo del reconocimiento maternal de la preñez, fue evaluado en 67 alpacas adultas receptivas al macho y que a la ecografía presentaban un folículo preovulatorio $\geq 7 \mathrm{~mm}$. Los animales se distribuyeron en 3 grupos: $\mathrm{G} 0(\mathrm{n}=23)$ como control; $\mathrm{G} 1(\mathrm{n}=22)$ que recibió $6 \mu \mathrm{g}$ GnRH el día 4 post-ovulación; y $\mathrm{G} 2(\mathrm{n}=22)$ que recibió $4 \mu \mathrm{g} \mathrm{GnRH}$ los días 8 y 9 postovulación. La ocurrencia de ovulación fue confirmada por la detección del cuerpo lúteo (CL) en el día 5 post-ovulación. La fertilización y viabilidad de la gestación fue monitoreada

\footnotetext{
${ }^{1}$ Práctica privada

${ }^{2}$ Laboratorio de Reproducción y Obstetricia Veterinaria, FMV-UNMSM

E-mail:vleyva@vet.unmsm.edu.pe

${ }^{3}$ Estación Experimental del Centro de Investigación IVITA-Maranganí, FMV-UNMSM
} 
por el desarrollo del CL, cambios estructurales de los cuernos uterinos y presencia de la vesícula embrionaria en los días $12,18,25$ y 30 post-ovulación por ecografía transrectal de los ovarios y útero. Simultáneamente se evaluó la receptividad sexual de la hembra al macho. El número de hembras que ovularon fue 20, 22 y 21 para G0, G1 y G2, respectivamente, considerándose que se encontraban gestantes (día 5); sin embargo, en el día 12 el porcentaje de supervivencia fue de $90.5 \%$ en G1, $75 \%$ en G0 y $76.2 \%$ en G2. El porcentaje de supervivencia embrionaria fue significativamente mayor en el día 25 y $30(\mathrm{p}<0.05)$ entre $\mathrm{G} 1(76.2 \%)$ y G2 (42.9\%). El diámetro folicular en G2 presentó una constante disminución desde el día 0 hacia los días 5 y 12 post-ovulación $(7.4,7.3$ y $6.3 \mathrm{~mm}$, respectivamente). El tamaño del CL en hembras que mantuvieron la gestación se incrementó desde el día 5 hacia los días 12 y 18 post-ovulación, mientras que los de hembras con mortalidad embrionaria presentaron una disminución significativa $(\mathrm{p}<0.01)$ desde el día 12 postovulación. El diámetro del CL y del folículo mostraron una relación inversamente proporcional con respecto a la mortalidad embrionaria. Se concluye que la GnRH disminuyó el tamaño del folículo dominante y la tasa de sobrevivencia embrionaria durante el periodo del reconocimiento maternal de la preñez.

Palabras clave: GnRH, alpaca, mortalidad embrionaria

\section{INTRODUCCIÓN}

Estudios en alpacas muestran que 30 a $40 \%$ requieren más de una monta para ovular (Sumar et al., 1993; Bravo et al., 1991) y que muchos problemas en la concepción son debidos a mortalidades embrionarias que ocurren en los primeros 35 días de la gestación (Fernández-Baca et al., 1970a). Estudios más recientes encontraron $20 \%$ de fallas ovulatorias por deficiente respuesta de la hembra al estímulo coital del macho (Leyva y García, 1999c) y 12\% de pérdida del óvulo fecundado dentro de los primeros 5 días postovulación (Leyva y García, 1999b); además, se sugirió que el estradiol de los folículos estrogénicos presentes alrededor de este periodo afectaban el desarrollo del cuerpo lúteo (Leyva y García, 2000). Estos mismos autores demostraron en base a la disminución del tamaño del cuerpo lúteo, que hay una relación entre cuerpos lúteos afectados y mortalidad embrionaria en estadíos posteriores (Leyva y García 1999b). La asociación de estos resultados con los de Fernández-Baca et al. (1970a) sugieren la ocurrencia de una mayor mortalidad en estadíos más avanzados de la gestación temprana; sin embargo, esta información requiere ser confirmada; sobretodo la magnitud de la mortalidad embrionaria y la probable alteración hormonal, alrededor del proceso del reconocimiento maternal de la preñez, el cual, en base a un descenso temporal de los niveles de progesterona, ha sugerido que puede ocurrir entre los días 9 y 11 post-ovulación en alpacas (Aba et al., 1995).

Frente a esta situación, Leyva y García (1999b) demostraron el efecto benéfico de la administración de GnRH en el día 5 post-servicio sobre la viabilidad de la fertilización y supervivencia embrionaria, la cual estimularía la hipófisis para la liberación de LH, y ésta a su vez, ejercería un efecto luteotrófico en el cuerpo lúteo para una mayor secreción de progesterona. El objetivo del presente estudio fue determinar si ocurre un efecto luteotrófico con el uso en la GnRH alrededor del periodo del reconocimiento maternal de la preñez para favorecer las tasas de supervivencia embrio-naria.

\section{Materiales y Métodos}

\section{Lugar de estudio y animales}

El experimento fue realizado en el Fundo San Luis (Provincia de Melgar, Puno), durante los meses de febrero a abril del 2001. 
El fundo, que pertenece a la Estación Experimental del Centro de Investigación IVITA-Maranganí, Facultad de Medicina Veterinaria de la Universidad Nacional Mayor de San Marcos, se encuentra a una altitud de 4,200 msnm.

Se seleccionaron 67 alpacas adultas hembras (>2 años) de la raza Huacaya. Los animales, que formaban parte del núcleo de reproductores, tenían un descanso sexual postparto >20 días, eran receptivas al macho y presentaban folículos preovulatorios $>7 \mathrm{~mm}$ de diámetro, determinados por ecografía transrectal ovárica, mediante un ecógrafo portátil B-mode Aloka 210 (Aloka Co., Led Tokio, Japón) equipado con un transductor lineal tipo array de $5 \mathrm{MHz}$ de frecuencia.

\section{Diseño experimental}

Las alpacas fueron servidas con macho fértil por el sistema de empadre controlado y la cópula tuvo una duración $\geq 15$ minutos (en algunos casos, las hembras tuvieron que ser cubiertas por dos machos para completar el tiempo de cópula establecido). Luego de la cópula, se les aplicó 500 UI de hCG (Intervet, Holland) vía i.m. para asegurar la ovulación.

Los animales fueron distribuidos al azar en los siguientes tratamientos:

- $\mathrm{G} 0$ : $(\mathrm{n}=23)$ grupo control ( $\sin \mathrm{GnRH})$

- G1: (n=22) Aplicación de $6 \mu \mathrm{g} \mathrm{GnRH}$ (Hoechst Roussel Vet.) en el día 4 post-ovulación

- G2: $(\mathrm{n}=22)$. Aplicación de $4 \mu \mathrm{g}$ de GnRH los días 8 y 9 post-ovulación

\section{Procedimiento metodológico}

El día 0 fue considerado el momento de la ovulación. El comportamiento sexual de la hembra (aceptación o rechazo al macho) se evaluó los días $5,12,18,25$ y 30 postovulación, previo a la observación ecográfica de los ovarios y útero, entre las 05:30 y las 07:00.
El estudio ecográfico de los ovarios y el útero permitió analizar la viabilidad de la fertilización, estimada por la presencia del cuerpo lúteo; así como la presencia de folículos estrogénicos $>5 \mathrm{~mm}$ de diámetro; los cambios estructurales del cuerno uterino grávido, y la presencia de la vesícula embrionaria y el embrión.

La tasa de ovulación, supervivencia embrionaria y concepción por grupos de tratamiento y la receptividad sexual en relación a la supervivencia embrionaria fueron evaluadas con la tabla de contingencia de Chi Cuadrado $\left(\chi^{2}\right)$. Diferencias en el tamaño del cuerpo lúteo y del folículo, y la supervivencia o mortalidad embrionaria en los diferentes estadíos, fueron evaluados por la prueba de análisis de varianza (ANOVA). Se utilizó la prueba de Tukey para establecer diferencias entre grupos.

\section{Resultados}

\section{Tasa de ovulación y supervivencia embrionaria}

Cuatro hembras ( 1 del G2 y 3 del Go) no llegaron a ovular y fueron excluidas del estudio. El porcentaje de supervivencia embrionaria en los animales que ovularon (Cuadro 1) fue mayor en $\mathrm{G}_{1}$ (90.5\%) que en G2 (76.2\%) y G0 (75.0\%) en el día 12 postovulación. Esta diferencia llegó a ser estadísticamente significativa $(\mathrm{p}<0.05)$ a partir del día 25 post-ovulación.

\section{Dinámica folicular}

El diámetro folicular promedio varió en el periodo post-ovulatorio bajo estudio, aunque estas diferencias sólo mostraron aproximación significativa $(\mathrm{p}<0.07)$ entre $\mathrm{G}_{\mathrm{o}}$ y $\mathrm{G} 2$ en el día 18 post-ovulación (Cuadro 2).

Independientemente de los tratamientos, las alpacas con mortalidad embrionaria detectada en el día 18 post-ovulación tuvieron folículos dominantes de mayor tamaño 
Cuadro 1. Porcentaje de ovulación y de supervivencia embrionaria en alpacas no tratadas $\left(\mathrm{G}_{0}\right)$, y tratadas con $\mathrm{GnRH}$ en el día $4\left(\mathrm{G}_{1}\right)$ y 8 y 9 post-ovulación $\left(\mathrm{G}_{2}\right)$

\begin{tabular}{lcccc}
\hline & $\mathrm{G}_{0}$ & $\mathrm{G}_{1}$ & $\mathrm{G}_{2}$ & Total \\
\hline Ovulación & & & & \\
$\mathrm{N}^{\mathrm{o}}$ de animales & 23 & 22 & 22 & 67 \\
$\mathrm{~N}^{\mathrm{o}}$ de animales con ovulación & 20 & 22 & 21 & 63 \\
$\mathrm{~N}^{\mathrm{o}}$ de ovulación & $87.0^{\mathrm{a}}$ & $100.0^{\mathrm{a}}$ & $95.5^{\mathrm{a}}$ & 94.0 \\
Porcentaje de supervivencia embrionaria & & & & \\
Día 5 post-ovulación & $100.0^{\mathrm{a}}$ & $100.0^{\mathrm{a}}$ & $100.0^{\mathrm{a}}$ & 100.0 \\
Día 12 post-ovulación & $75.0^{\mathrm{a}}$ & $90.5^{\mathrm{a}}$ & $76.2^{\mathrm{a}}$ & 80.7 \\
Día 18 post-ovulación & $55.0^{\mathrm{a}}$ & $81.0^{\mathrm{a}}$ & $66.7^{\mathrm{a}}$ & 67.7 \\
Día 25 post-ovulación & $55.0^{\mathrm{ab}}$ & $76.2^{\mathrm{b}}$ & $42.9^{\mathrm{ac}}$ & 58.1 \\
Día 30 post-ovulación & $55.0^{\mathrm{ab}}$ & $76.2^{\mathrm{b}}$ & $42.9^{\mathrm{ac}}$ & 58.1 \\
\hline
\end{tabular}

abc Valores con letras diferentes en una misma fila son significativamente diferentes $(p<0.05)$

Cuadro 2. Tamaño folicular promedio $\left(\mathrm{mm} \pm\right.$ d.e) en alpacas no tratadas $\left(\mathrm{G}_{0}\right)$, y tratadas con $\mathrm{GnRH}$ en el día $4\left(\mathrm{G}_{1}\right)$ y 8 y 9 post-ovulación $\left(\mathrm{G}_{2}\right)$

\begin{tabular}{cccc}
\hline Día post-ovulatorio & $\mathrm{G}_{0}$ & $\mathrm{G}_{1}$ & $\mathrm{G}_{2}$ \\
\hline (Servicio) & $7.7 \pm 1.8$ & $7.5 \pm 1.3$ & $7.4 \pm 1.1$ \\
5 & $6.2 \pm 2.7$ & $5.7 \pm 1.5$ & $7.3 \pm 2.6$ \\
12 & $7.0 \pm 1.7$ & $6.7 \pm 1.9$ & $6.3 \pm 1.1$ \\
18 & $8.0 \pm 1.7$ & $6.6 \pm 2.7$ & $6.1 \pm 1.7$ \\
\hline
\end{tabular}

$(7.8 \pm 1.7 \mathrm{~mm})$ que alpacas con supervivencia embrionaria $(5.3 \pm 1.9 \mathrm{~mm}, \mathrm{p}<0.01)$.

\section{Relación entre cuerpo lúteo y folículo}

El tamaño del cuerpo lúteo y del folículo están en una relación inversamente proporcional en hembras con supervivencia embrionaria, mientras que ocurre en forma contraria en animales con mortalidad embrionaria (Fig. 1).

\section{Cuerpo lúteo en relación a la supervi- vencia embrionaria}

El tamaño del cuerpo lúteo en los días 12 y 18 post-ovulación fue estadísticamente diferente en las alpacas hembras con supervivencia embrionaria $(13.3 \pm 1.3$ y $13.7 \pm 1.7$ $\mathrm{mm}$, respectivamente) que en las alpacas que tuvieron mortalidad embrionaria (10.3 \pm 1.0 y $9.9 \pm 1.2 \mathrm{~mm}$, respectivamente). 


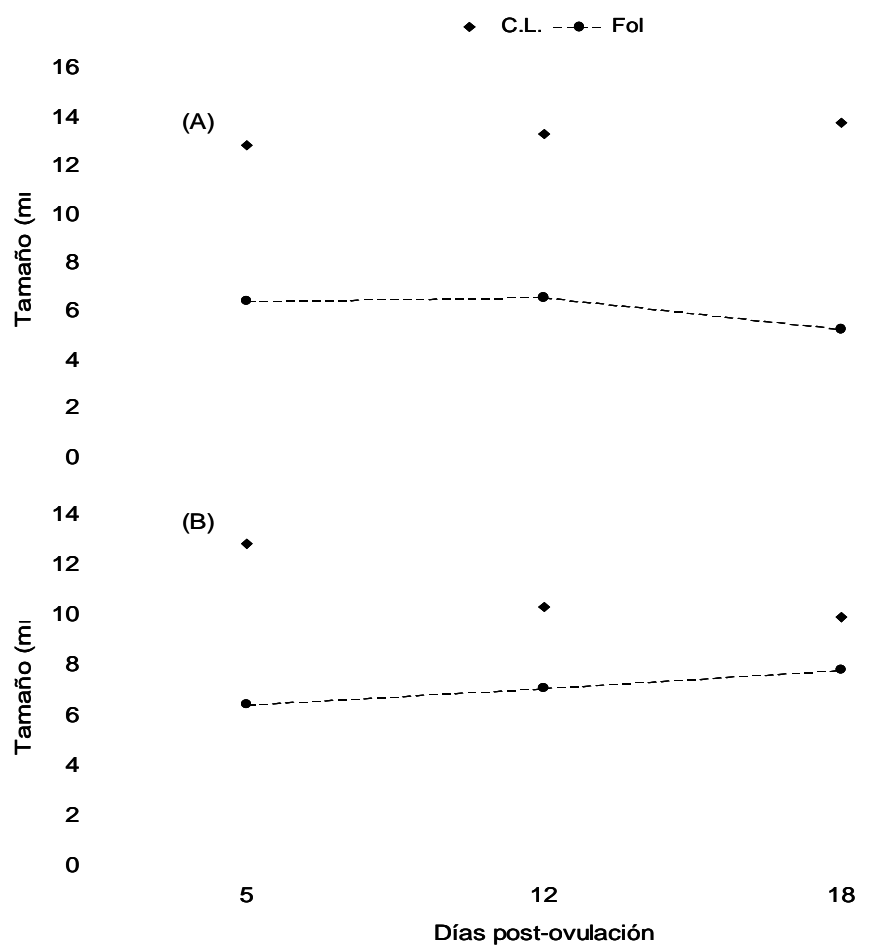

Fig. 1. Relación entre cuerpo lúteo $(\mathrm{CL})$ y folículo (Fol) en hembras con supervivencia (A) y mortalidad embrionaria (B)

Cuadro 3. Receptividad sexual en alpacas gestantes y no gestantes en diferentes periodos de desarrollo del cuerpo lúteo

\begin{tabular}{cccccc}
\hline \multirow{2}{*}{ Día post-ovulación } & \multicolumn{2}{c}{ Hembras gestantes } & & \multicolumn{2}{c}{ Hembras no gestantes } \\
\cline { 2 - 3 } \cline { 5 - 6 } & Acepta $(\%)$ & Rechaza $(\%)$ & & Acepta $(\%)$ & Rechaza $(\%)$ \\
\hline 5 & $19.1^{\mathrm{a}}$ & $81.0^{\mathrm{b}}$ & & - & - \\
12 & $12.0^{\mathrm{a}}$ & $88.0^{\mathrm{b}}$ & & $83.3^{\mathrm{a}}$ & $16.7^{\mathrm{b}}$ \\
18 & $14.3^{\mathrm{a}}$ & $85.7^{\mathrm{b}}$ & & $100.0^{\mathrm{a}}$ & $0.0^{\mathrm{c}}$ \\
25 & $2.9^{\mathrm{c}}$ & $97.1^{\mathrm{b}}$ & & $96.3^{\mathrm{a}}$ & $3.7^{\mathrm{c}}$ \\
30 & $2.9^{\mathrm{c}}$ & $97.1^{\mathrm{b}}$ & & $100.0^{\mathrm{a}}$ & $0.0^{\mathrm{c}}$ \\
\hline
\end{tabular}

a,b,c Valores con letra diferente dentro de cada grupo (gestante y no gestante) son significativamente diferentes $(p<0.05)$

\section{Receptividad sexual}

El comportamiento de receptividad sexual en relación a la supervivencia o mortalidad embrionaria en alpacas en diferentes periodos se aprecia en el Cuadro 3. Se ob- servó una asociación marcada de hembras que mantienen el cuerpo lúteo (supervivencia embrionaria) con la no receptividad al macho (rechazo), mientras que las hembras que sufrieron mortalidad embrionaria mostraron una clara receptividad al macho (aceptación). 


\section{Discusión}

Se asume que el periodo del reconocimiento maternal de la preñez en alpacas ocurre entre los días 9 y 10 post-ovulación (10 y 11 post-servicio), dado que entre estos días ocurre una disminución temporal de los niveles de progesterona en las alpacas gestantes (Aba et al., 1995; Aba et al., 1997). También, en este periodo es cuando ocurre la luteólisis en las alpacas vacías (FernándezBaca et al., 1970b; Leyva y García, 1999a).

En el presente estudio los animales del grupo que recibieron GnRH en los días 8 y 9 post-ovulación $\left(\mathrm{G}_{2}\right)$ tuvieron menor tasa de supervivencia embrionaria y una disminución en el tamaño del folículo dominante desde el día 5 hasta el día 12 post-ovulación; lo cual contrasta con los resultados obtenidos en vacunos por Mann et al. (1995) y Sheldon y Dobson (1993) que obtienen una mayor tasa de supervivencia por la administración de GnRH entre los días 11 y 13 post-ovulación, próximos al periodo del reconocimiento maternal de la preñez. Estos autores encontraron una disminución en el tamaño del folículo dominante e indirectamente sugirieron que hubo interferencia con la síntesis de los receptores de oxitocina como un medio para bloquear la luteólisis del cuerpo lúteo.

El grupo de alpacas que recibió $\mathrm{GnRH}$ en el día 4 post-ovulación tuvo la mayor tasa de supervivencia embrionaria, lo cual concuerda y da soporte a los resultados obtenidos por Leyva y García (1999b), que infieren un efecto luteotrópico de la GnRH sobre la actividad secretoria de la progesterona para producir un efecto inhibitorio a nivel del hipotálamo en la supresión del celo (Leyva y García, 1999a.; Santiani, 2001) y a nivel ovárico inhibiendo el desarrollo folicular (Santiani, 2001; Vaughan, 2001), e indirectamente en la producción de estradiol, interfiriendo de esta manera con la síntesis de receptores de oxitocina evitando la muerte prematura del cuerpo lúteo (Leyva y García, 1999b.; Leyva y García, 2000). En el presen- te estudio hubo una disminución en el tamaño folicular entre el día 0 (7.5 $\mathrm{mm}$ de diámetro) y el día 5 (5.6 mm de diámetro) en el grupo tratado con GnRH en el día 4 post-ovulación; mientras que en el grupo con mayor mortalidad embrionaria no hubo disminución en el tamaño folicular $(7.4 \mathrm{~mm}$ en el día 0 y 7.3 $\mathrm{mm}$ en el día 5 post-ovulación). Estos resultados dan mayor soporte a la hipótesis de Leyva y García (1999b) quienes infieren que los días 4 y 5 post-ovulación son críticos en la supervivencia del cuerpo lúteo por la presencia del folículo estrogénico; cuyo efecto sobre la mortalidad embrionaria y del cuerpo lúteo fue reportado posteriormente (Leyva y García, 2000).

En condiciones normales, la alpaca manifiesta aceptación al macho hasta el día 5 post-servicio porque los niveles de progesterona secretada por el cuerpo lúteo son aún insuficientes para ejercer un efecto inhibitorio al celo (Leyva y García, 1999a). No obstante, en este estudio, hubo un número importante de hembras en gestación con presencia de cuerpo lúteo que aceptaron al macho. Similar comportamiento también fue reportado por Aparicio (2001), sugiriendo que este comportamiento manifiesto sea el resultado de un acondicionamiento de las hembras, como consecuencia del manejo reproductivo establecido en el Centro Experimental del estudio o también probablemente por fallas en la interpretación del comportamiento sexual.

\section{Conclusiones}

- La aplicación de la GnRH durante los días 8 y 9 post-ovulación disminuyó la tasa de supervivencia embrionaria y el tamaño del folículo dominante.

- La aplicación de la GnRH en el día 4 postovulación mejoró la tasa de supervivencia embrionaria.

- Las alpacas con supervivencia embrionaria tuvieron menor tamaño folicular el día 5 post-ovulación y mayor tamaño en el día 12 post-ovulación. 
- El incremento en tamaño del cuerpo lúteo se relaciona con una disminución en el tamaño folicular.

- Un $14 \%$ de hembras gestantes fueron sexualmente receptivas hasta el día 18 post-ovulación.

\section{Literatura Citada}

1. Aba, M.A.; M. Forsberg; H. Kindahl; J. Sumar; L.E. Edqvist. 1995. Endocrine changes after mating in pregnant and non-pregnant llamas and alpacas. Acta Vet. Scand. 36: 489-498.

2. Aba, M.A.; P.W. Bravo; M. Forsberg; H. Kindahl. 1997. Endocrine changes during early pregnancy in the alpaca. Anim. Reprod. Sci. 47: 273-279.

3. Aparicio, M. 2001. Efecto de la frecuencia de copulación en alpacas durante el celo postovulatorio sobre la mortalidad embrionaria. Tesis de Médico Veterinario. Facultad de Medicina Veterinaria, Univ. Nacional Mayor de San Marcos, Lima. 44 p.

4. Bravo, P.W.; G.H. Stabenfeldt; B.L. Lasley; M.E. Fowler. 1991. The effect of ovarian follicle size on pituitary and ovarian responses to copulation in domesticated South American camelids. Biol. Reprod. 45: 553-559.

5. Fernández-Baca, S.; W. Hansel; C. Novoa. 1970a. Embryonic mortality in the alpaca. Biol. Reprod. Fertil. 3: 243251.

6. Fernández-Baca, S.; W. Hansel; C. Novoa. 1970b. Corpus luteum function in the alpaca. Biol. Reprod. Fertil. 3: 252261.

7. Leyva, V.; W. García. 1999a. Efecto de la progesterona exógena sobre la fun- ción del cuerpo lúteo de alpacas. En: Res. II Cong. Mund. Camélidos. Cusco. p 87.

8. Leyva, V.; W. García, 1999b. Efecto de la GnRH sobre la fertilización y sobrevivencia embrionaria en alpacas. En: Res. II Cong. Mund. Camélidos. Cusco. p 90.

9. Leyva, V.; W. García, 1999c. Efecto de la progesterona exógena y endógena en alpacas en celo sobre la ovulación, fertilización y gestación. En: Res. II Cong. Mund. Camélidos. Cusco. p 89.

10. Leyva, V.; W. García. 2000. Efecto del estradiol (E2) en la fertilización y sobrevivencia embrionaria en alpacas. En: Res. XV Cong. Nac. Cienc. Vet. Cusco. $\mathrm{p} 22$.

11. Mann, G.E.; G.E. Lamming; M.D. Fray. 1995. Plasma oestradiol and progesterone during early pregnancy in the cow and the effects of treatment with buserelin. Anim. Reprod. Sci. 37: 121131.

12. Santiani, A. 2001. Efecto inhibitorio de la progesterona sobre el desarrollo de la onda folicular en llamas. Tesis de Médico Veterinario. Facultad de Medicina Veterinaria, Univ. Nacional Mayor San Marcos, Lima. 48 p.

13. Sheldon, I.M.; H. Dobson. 1993. Effects of gonadotropin releasing hormone administered 11 days after insemination on the pregnancy rates of cattle to the first and later services. Vet. Rec. 133: 160-163.

14.Sumar, J.; W. Bravo; W.C. Foote. 1993. Sexual receptivity and time of ovulation in alpacas. Small Rum. Res. 11: 143-150.

15. Vaughan, J. 2001. Control of follicular waves in alpacas. Rev. Inv. Vet. Perú Supl. 1: 112-114. 